\title{
Gait characteristics of CKD patients: a systematic review
}

Damiano D. Zemp ${ }^{1,2}$, Olivier Giannini $i^{3,4,6}$, Pierluigi Quadri ${ }^{2,3}$ and Eling D. de Bruin ${ }^{1,5^{*}}$ (1)

\begin{abstract}
Background: People with Chronic Kidney Disease (CKD) often present with prevalent gait impairment and high fall rates, particularly in advanced CKD stages. Gait impairment and its consequences is associated with increased hospital admission, institutionalization, and greater need for health care. The objective of this systematic review was to evaluate the quality of studies investigating CKD patients' gait characteristics at different CKD stages, to highlight areas of agreement and contradiction between studies reporting aspects of gait in CKD, and to discuss and emphasize gait parameters associated with fall risk.

Methods: We performed a literature search of trials in CINAHL (EBSCO), Cochrane Library, EMBASE, Medline (EBSCO) , PEDro, PubMed, and Scopus databases from their inception to June 30th 2018 using a two-stage process for the identification of studies. We retrieved English-, German-, Italian-, Spanish-, Portuguese and Dutch-language articles for review. Methodological quality of randomized and non-randomized studies was assessed with an adapted version of the Downs and Black checklist.

Results: Thirty-one studies (22 cross-sectional with 3901 participants) and 9 longitudinal intervention studies (1 randomized control trial, 5 controlled clinical trials and 3 one-group pre-post-test; with 659 participants) were considered. The studies revealed a primary emphasis on gait speed measures within clinical tests, and a neglect of spatiotemporal gait variables. Most of the studies showed that CKD progression is associated with slowing of walking speed. No studies analysed the relation between gait parameters and fall risk.

Conclusions: There was a paucity of studies investigating aspects of gait quality in patients with CKD. In the majority of studies, only gait speed is analysed as a performance indicator. The relation between gait parameters and fall risk in CKD is not investigated. We formulate several recommendations to fill the current research gap, encourage the use of standardized gait analysis protocols that include assessment of spatiotemporal parameters in clinical care of patients with CKD, aimed at prevention of mobility decline and falls risk.
\end{abstract}

Keywords: Chronic Kidney disease, Gait, Mobility, Falls

\section{Background}

Chronic Kidney Disease (CKD) is defined as abnormalities in kidney structure or function, present for 3 months, with implications for health [1]. CKD is classified in stages 1 to 5 , with higher stages representing lower glomerular filtration rate levels [2]. A potential outcome of CKD is end-stage renal disease (ESRD),

\footnotetext{
* Correspondence: eling.debruin@hest.ethz.ch

${ }^{1}$ Institute of Human Movement Sciences and Sport, Department of Health Sciences and Technology, ETH Zurich, HCP H 25.1, Leopold-Ruzicka-Weg 4, Zurich, Switzerland

${ }^{5}$ Division of Physiotherapy, Department of Neurobiology, Care Sciences and Society, Karolinska Institute, Stockholm, Sweden

Full list of author information is available at the end of the article
}

requiring costly renal replacement therapy in the form of dialysis or transplantation. CKD ranks 11th place among the leading causes of death globally [3], exhibits unfavourable trends in age-standardized death and disability-adjusted life years (DALYs) rates [4], and its prevalence worldwide is estimated to be $8-16 \%$ [5].

Falls in elderly patients on dialysis are common. The falls rate in this population lies between 38 and 47\% [6, 7], whereas $1 / 3$ of the senior population not on dialysis fall at least once a year [8]. Recent studies show higher fall rates and fracture risk already in CKD patients not on dialysis $[9,10]$. Results from a retrospective cohort analysis seems to indicate, however, that dialysis therapy initiation may

(c) The Author(s). 2019 Open Access This article is distributed under the terms of the Creative Commons Attribution 4.0 International License (http://creativecommons.org/licenses/by/4.0/), which permits unrestricted use, distribution, and 
be a precipitating factor for falls [11], which might explain in part the higher fall rates seen in this population.

In clinical geriatric practice, instrumental gait analysis [12], often combined with functional tests [13] and instrumental posture analysis [14], is used widely for risk of falls assessment. In this regard, gait assessment considering both physical and cognitive aspects is meaningful because gait requires both kinds of resources $[15,16]$. However, conflicting information can be found in the literature about gait in CKD. Where some attribute changes in walking speed to the presence of chronic kidney disease [17], other sources state that these observed changes are due to diseases accompanying some of the CKD patients; e.g. diabetes with peripheral neuropathy [18]. Therefore, a better understanding of gait disorders in CKD patients and the progression of these impairments with increasing CKD severity could be useful to quantify fall risk in this population, and might help identify possible amenable parameters for preventive interventions.

Although there are suggestions that patients show gait abnormalities already in early stages of CKD that lead to heightened risk of falling, little is known about which gait parameters at which stages of CKD could be clinically relevant for fall risk prevention. This systematic review, therefore, focuses on gait characteristics in various stages of CKD and on understanding their possible relation to falls as reported in the scientific literature. We aim in particular to: (1) evaluate the quality of existing studies investigating CKD patients' gait characteristics at different CKD stages, (2) highlight areas of agreement and contradiction in study results, and (3) analyse the association between gait parameters and falls risk.

\section{Methods}

In March 2017, with an upgrade in July 2018, a professional librarian performed an electronic search of electronic databases from their inception to 2018/06/30 (CINAHL (EBSCO), Cochrane Library, EMBASE, Medline (EBSCO), PEDro, PubMed, Scopus), using a two-stage process for the identification of potential studies.

We combined free-text and Medical Subject Headings terms using a broad range of synonyms, related terms and variant spelling. Second, all reference lists of review articles and included articles were scanned manually. We retrieved English-, German-, Italian-, Spanish-, Portuguese and Dutch-language articles for review.

Three semantic search loops were used (Additional file 1). The first loop contained terms related to kidney disease, the second related to gait and the third included key words that relate to falls. For the identification of relevant falls risk factors, we used two previously published systematic reviews with meta-analyses $[19,20]$.

\section{First selection based on abstracts}

Two independent reviewers (DZ, EdB) assessed the titles, the abstracts and, where necessary, full texts, to determine the eligibility of each article. Cross-sectional, randomised control trials and controlled clinical trials of human studies were included when (a) a kidney disorder is mentioned; (b) at least one quantitative gait related parameter was measured (e.g. as part of gait analysis or a functional test such as the Short Physical Performance Battery or the Timed Up \& Go Test); (c) in English, German, Italian, French, Spanish, Portuguese or Dutch. Studies were excluded when published in an abstract form only. Disagreements between reviewers were resolved by consensus-finding discussion. PRISMA (Preferred Reporting Items for Systematic Reviews and Meta-Analysis) guidelines were followed to ensure the clarity and transparency of reporting of this systematic review [21, 22] (Fig. 1).

\section{Data extraction}

From the eligible manuscripts, the CKD stage, the study population (age, Body Mass Index (BMI), sex), applied selection criteria, gait test, and measured gait parameters, were extracted.

Data were extracted using Microsoft Excel templates purpose-developed for this review. Mean and standard deviation of age, BMI and gait speed in $\mathrm{m} / \mathrm{s}$ over the different studies were calculated, and the studies were weighted on the basis of the reported number of their participants. Because original individual data were not available no formal statistical comparison to determine whether differences between CKD groups were apparent was possible.

\section{Quality analysis}

Quality of the included articles was assessed by two independent reviewers (DZ, EdB) using Downs and Black checklist for randomized and non-randomized trials [23], which was developed for the assessment of the methodological quality of both randomized and non-randomized studies. We adapted the checklist, discarding redundant items 4, 8, 9 and 17 for cross-sectional studies ([18, 2444]), and items 14 and 15 for all studies. Item 23 and 24 were considered only for randomized control trials ([45]) and controlled clinical trials ([46-50]) studies. Power (item 27) was considered only in longitudinal intervention studies ([45-53]) and in cross-sectional studies involving a control group ([25, 28, 33, 34, 41-43]). Items 5 and 27 were modified and scored 0 or 1 instead of $0-2$ and $0-5$ respectively [54]. Adapting the checklist, maximum achievable points differed from one study to another. Therefore, quality score was calculated as the ratio of achieved points to maximum points, indicating 0 for poor 


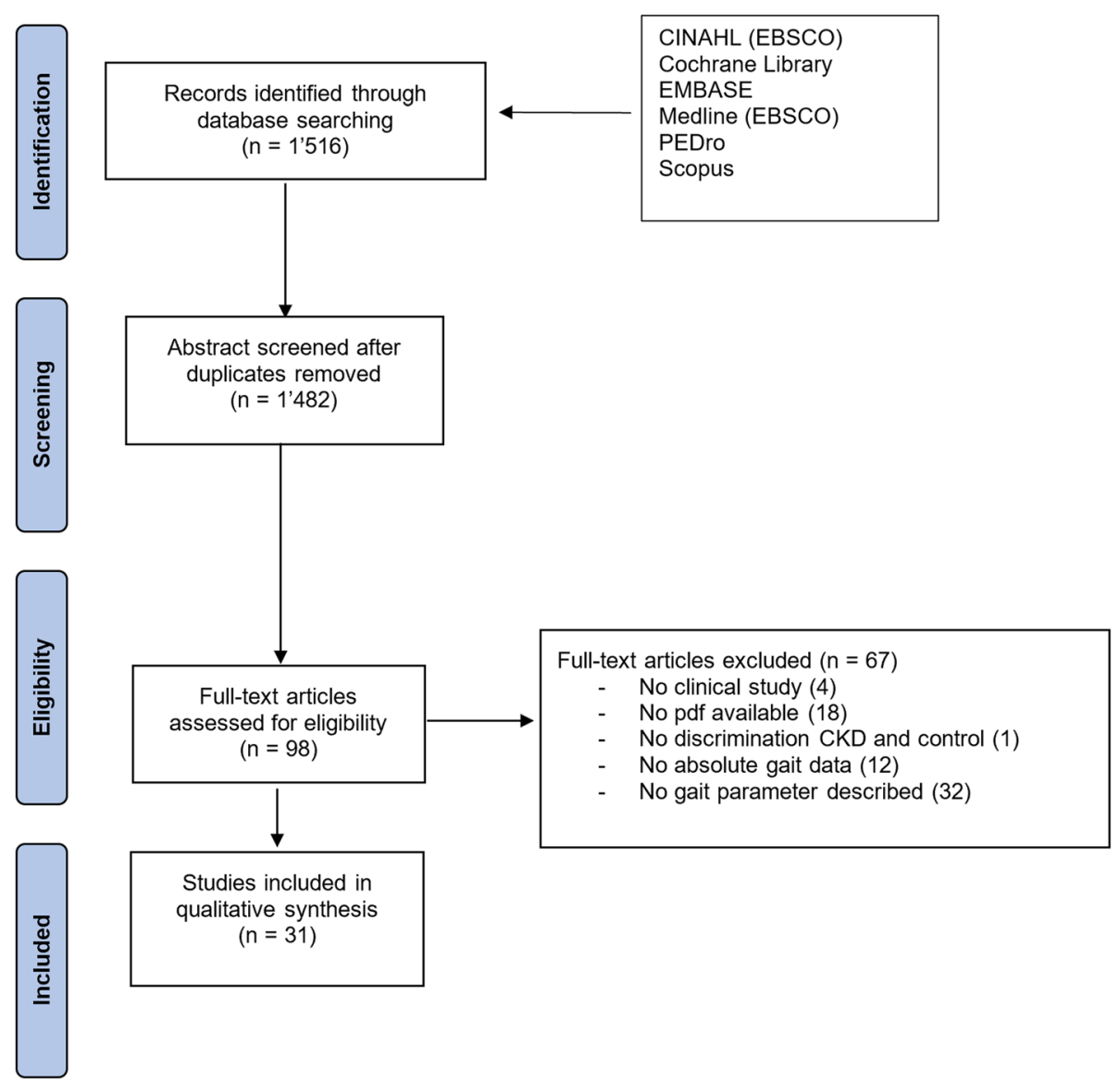

Fig. 1 PRISMA flow chart of the articles' selection process

and 1 for high quality of the study respectively for each sub-category evaluated.

Kappa statistics was used to calculate inter-rater agreement and interpreted in accordance with Landis and Koch's benchmarks [55] for assessing the agreement between reviewers: poor $(<0.00)$, slight $(0.00-0.20)$, fair (0.21-0.40), moderate $(0.41-0.60)$, substantial $(0.61-0.80)$, and almost perfect $(0.81-1.00)$.

\section{Results}

\section{First selection based on abstracts}

Database searching resulted in 1516 articles. After removing duplicates and screening of titles, abstracts and full texts following inclusion criteria, 31 articles with 4560 patients were selected for the review. A summary is given in Fig. 1 and Table 1.

\section{Data quality}

The agreement on data quality between the two reviewers was high. The estimated Kappa value was $0.81 \pm 0.02$. The $95 \%$ confidence interval ranged from 0.77 to 0.86 . The quality scores of studies ranged from 0.28 to 1.00 . The mean quality was $0.50 \pm 0.15$. The mean score was $0.76 \pm 0.16$ for reporting, $0.19 \pm 0.25$ for external validity, $0.58 \pm 0.22$ for internal validity bias, $0.12 \pm 0.24$ for internal validity, and $0.28 \pm 0.46$ for power. The results of quality assessment are summarized in Additional file 2.

\section{Gait assessment protocols}

In all included articles, the gait test assessment consisted of a timed walk during a defined walking course distance. Two publications reported use of a pressure sensitive walkway system (Gaitrite ${ }^{\circ}$ ) that allows more detailed gait analysis; e.g. spatiotemporal gait parameters and their variability $[42,43]$.

The walk test distance used ranged between $4 \mathrm{~m}$ $[27,28,38,41]$ and $50 \mathrm{ft} .(15.2 \mathrm{~m})$ [32, 33]. The most used distances were $20 \mathrm{ft}$. $(6.1 \mathrm{~m})$ in 10 articles [25, $26,29,34,39,45,48,49,51,53]$ and $10 \mathrm{~m}$ in 6 articles $[24,30,31,37,46,50]$. Whereas gait distance was reported in meters or in feet, speed was always measured using international units $\mathrm{m} / \mathrm{s}$ or $\mathrm{cm} / \mathrm{s}$.

In 11 studies, people performed a standing start $[18,27,28,31,39-41,45,47,48,50]$, so acceleration is therefore included in the speed calculation. In 7 manuscripts, a flying start excluding acceleration was 


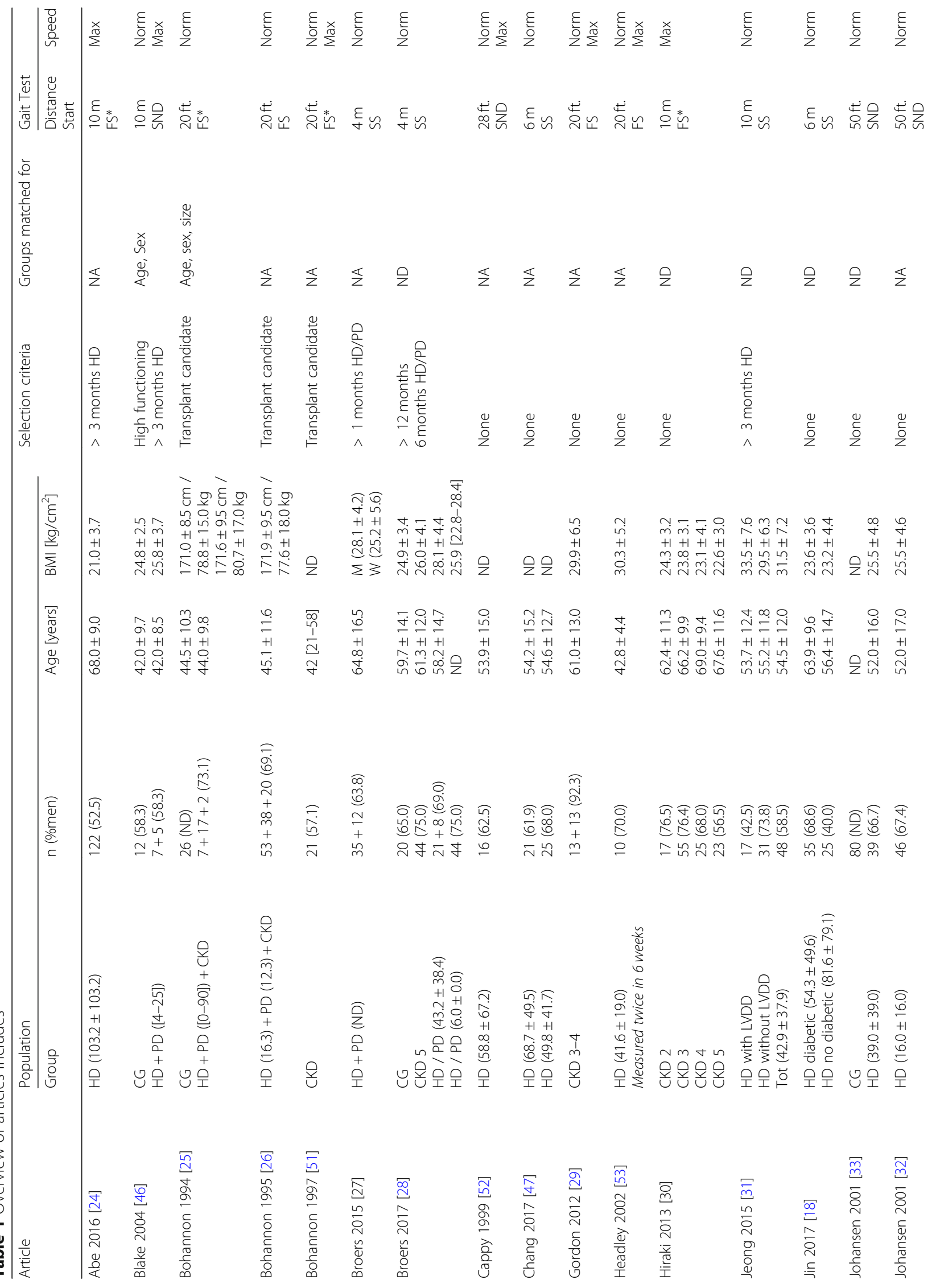




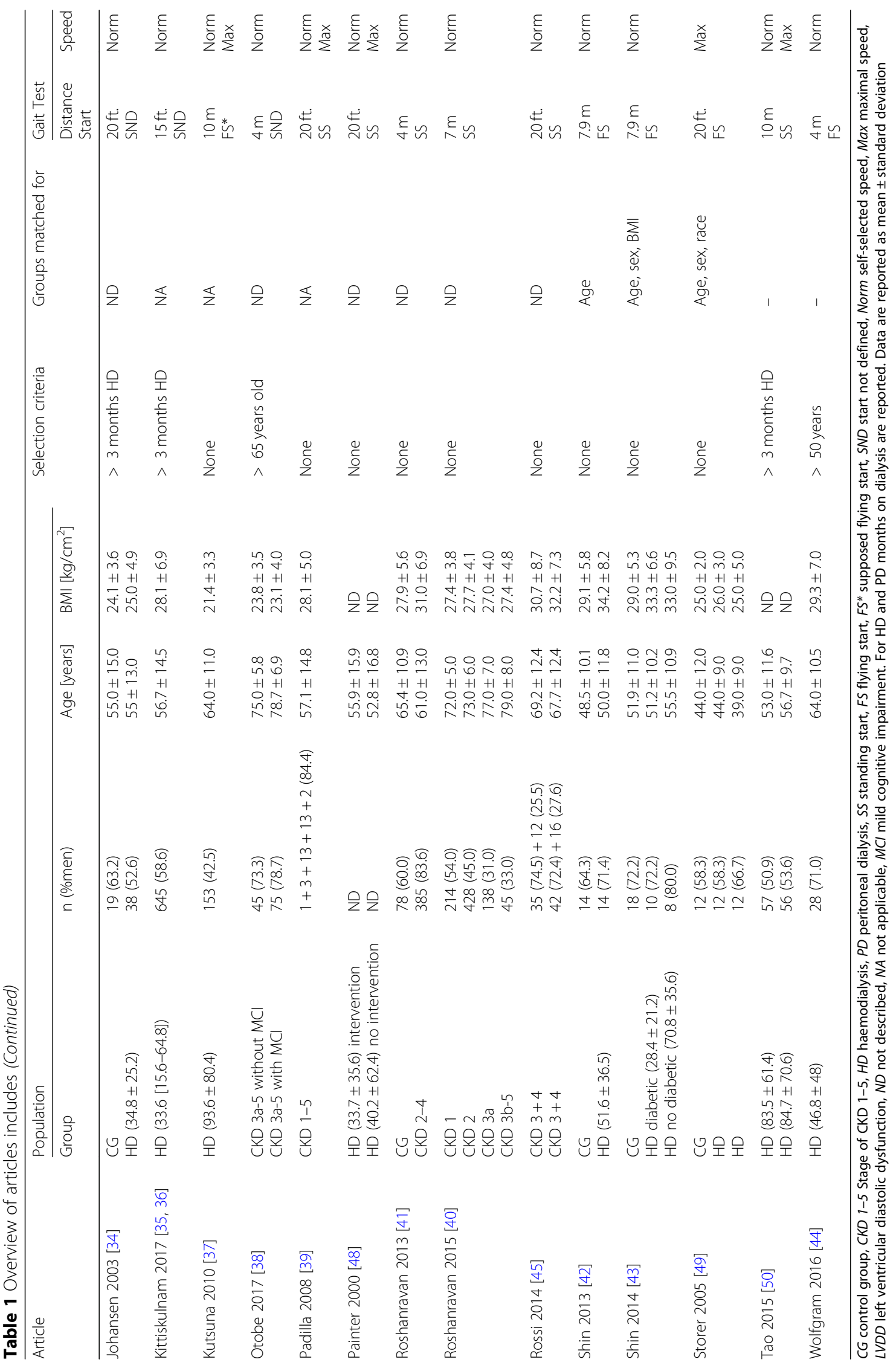


reported [26, 29, 42-44, 49, 53]. Thirteen studies failed to describe clearly the start procedure [24, 25, $30,32-38,46,51,52]$; however, in 5 cases $[24,25$, $30,37,51]$ we could assume a flying start was used because the authors made reference to other publications using such protocols. Due to the lack of information in many studies about the starting procedure and no apparently significant speed difference in studies using a flying or a standing start, this criterion was not considered in calculating the mean values of each CKD group. In 28 studies participants were asked to walk at a self-selected speed [18, 25-29, 31$48,50-53]$. In 9 of these studies [29, 37, 39, 46, 48, 50-53], people additionally walked at their maximal speed. In one case people walked at self-selected speed under a single- and a dual-task test condition [42], thus considering cognitive contributions to gait [56]. Three protocols measured people at their maximal speed only [24, 30, 49]. The most measured gait parameter was gait speed. Twenty-four of 27 studies only measured this outcome parameter for gait. One publication measured stride time in addition [46]. The two studies that used the sensitive walkway system, measured, along with gait speed and cadence, also the mean and coefficient of variation of stride length, stride time and width; of swing and stance time and of base-of-support and percent of double-support within the gait cycle [42, 43]. An overview of reported gait assessment protocols of the studies is listed in Table 1.

\section{Population}

The mean age of 4560 participants was 61.6 years [42-79], with 23 groups in average older and 37 younger than 60 years. $61.5 \%$ were male [31-92\%] and the mean BMI was $27.3 \mathrm{~kg} / \mathrm{m}^{2}$ [21-34]. In 31 publications, we found clinical gait assessment performed in 51 groups of CKD patients and in 9 control groups with 279 participants. Eight groups consisted of patients at a specific CKD stage (one for stage 1 with 214 patients, two each for stages 2 (445 patients) and 3 (193 patients), one for stage 4 (25 patients), and two for stage 5 not on dialysis with 67 patients). The most analysed population was dialysis patients (28 groups with 1673 patients). Fifteen groups with 760 patients where heterogeneous and included patients at different CKD stages.

One publication reported on high functioning HD patients (low comorbidity and self-perceived well-functioning) [46], two compared haemodialysis (HD) patients with and without diabetes $[18,43]$, one compared HD patients with and without left ventricular diastolic dysfunction (LVDD) [31], two CKD patients with and without diabetes $[25,26]$ and one CKD patients with and without Mild Cognitive Impairment (MCI) [38]. The remaining publications did not mention exclusion or inclusion criteria, apart from the presence of CKD.

A list of summary descriptions of participants is given in Table 1.

\section{Gait characteristics}

Mean gait speed at self-selected and maximal speed in dialysis patients is lower when compared to control groups and to CKD patients not on dialysis. Mean self-selected gait speed in dialysis groups was between 0.71 and 1.70 , with a mean gait speed of $1.12 \mathrm{~m} / \mathrm{s} \mathrm{com-}$ pared to $1.41 \mathrm{~m} / \mathrm{s}$ [1.31-1.80] in controls. Maximal gait speed ranged in dialysis groups between 1.47 and 1.86 $\mathrm{m} / \mathrm{s}$ (mean 1.57), whereas control groups walked maximal at $2.16 \mathrm{~m} / \mathrm{s}$ [1.89-2.29]. CKD patients not on dialysis have slower self-selected gait speed than controls, which decreases with advancing CKD severity (1.25 \pm $0.24 \mathrm{~m} / \mathrm{s}$ in CKD stage $1,1.19 \pm 0.25 \mathrm{~m} / \mathrm{s}$ in CKD stage 2 and $1.06 \pm 0.28 \mathrm{~m} / \mathrm{s}$ in CKD stage 3$)$. The same is observed for maximal gait speed, but only up from CKD stages $3(2.10 \pm 0.40 \mathrm{~m} / \mathrm{s}$ in CKD $3,1.70 \pm 0.50 \mathrm{~m} / \mathrm{s}$ in CKD 4 and $1.70 \pm 0.40 \mathrm{~m} / \mathrm{s}$ in CKD 5). Although studies with patients on dialysis recorded the modality of replacement therapy, no statistical analysis based on dialysis modality was found. Patients that suffer, along with CKD, from diabetes or LVDD showed generally slower gait speed compared to a matched group free of the second disorder. All gait speed data are summarized in Table 2. One study [28] measured in a $4 \mathrm{~m}$ walk test, at self-selected speed, a much higher gait speed when compared to all the other publications: $1.8 \mathrm{~m} / \mathrm{s}$ for controls, $1.4 \mathrm{~m} / \mathrm{s}$ for CKD stage 5 , and $1.5 \mathrm{~m} / \mathrm{s}$, for dialysis patients. Another author [44] reported in HD patients, performing a $4 \mathrm{~m}$ walk test at self-selected speed, much slower speed $(0.76 \mathrm{~m} / \mathrm{s})$ compared to all other HD studies. A detailed description of gait parameters of all studies is available in Additional file 3.

Stride time was found to be higher in HD-patients compared to controls $[43,46]$. The same was found for step time both under single- and dual-task conditions [42].

The complete gait assessment made using a pressure sensitive walkway system (Gaitrite ${ }^{\circledast}$ ) showed a much more conservative gait pattern of HD patients compared to controls, with slower gait speed, shorter stride length and longer double-support phase. The difference is much higher in diabetic HD-patients compared to non-diabetic individuals. The HD group showed also a higher stride variability and a higher dual-task cost, especially for gait speed and cadence, when compared to controls $[42,43]$.

\section{Falls related gait parameters}

No studies analysed the relation between gait parameters and fall risk. 
Table 2 Gait parameters measured in the 51 groups analysed

\begin{tabular}{lllll}
\hline Population & Gait speed $(\mathrm{m} / \mathrm{s})$ & & & Stride time $(\mathrm{s})$ \\
\cline { 2 - 4 } & Self-selected & Maximal & Self-selected under dual-task condition & $1.28 \pm 0.22$ \\
\hline Control $(n=9)$ & $\begin{array}{l}1.41[1.31-1.80] \\
n=9 / 9\end{array}$ & $\begin{array}{l}2.16[1.89-2.29] \\
n=4 / 9\end{array}$ & $n=1 / 9$ & $n=3 / 9$ \\
CKD 1 $(n=1)$ & $1.25 \pm 0.24$ & & \\
& $n=1 / 1$ & & \\
CKD 2 & $1.19 \pm 0.25$ & $2.20 \pm 0.20$ & \\
$(n=2)$ & $n=1 / 2$ & $n=1 / 2$ & \\
CKD 3 & $1.06 \pm 0.28$ & $2.10 \pm 0.40$ & \\
$(n=2)$ & $n=1 / 2$ & $n=1 / 2$ & \\
CKD 4 & & $1.70 \pm 0.50$ & \\
$(n=1)$ & $n=1 / 1$ & \\
CKD 5 & $1.40[1.2-1.8]$ & $1.70 \pm 0.40$ & $n=1 / 2$ & \\
$(n=2)$ & $n=1 / 2$ & $1.57[1.47-1.86]$ & $0.87 \pm 0.25$ \\
Dialysis & $1.12[0.71-1.70]$ & $n=9 / 28$ & $n=1 / 28$ \\
$(n=28)$ & $n=26 / 28$ & $1.2[1.04-1.31]$ \\
\hline
\end{tabular}

Overall mean and [range] in case of $>1$ group or \pm SD if only 1 group is described. $n=$ number of groups included

anot on dialysis

\section{Intervention studies}

Eight studies investigated the effect of different interventions on gait in CKD patients (Table 3). Three studies showed that HD patients can improve self-selected and maximal speed through regular physical activity including flexibility, strengthening and cardiovascular training [50, 52, 57]. Strength training [53] in HD patients and

Table 3 Intervention studies

\begin{tabular}{|c|c|c|c|}
\hline Authors & Intervention & Improvement & Population \\
\hline $\begin{array}{l}\text { Bohannon } \\
1997 \text { [51] }\end{array}$ & $\begin{array}{l}\text { Kidney } \\
\text { transplantation. }\end{array}$ & Maximal gait speed. & CKD \\
\hline $\begin{array}{l}\text { Cappy } \\
1999[52]\end{array}$ & $\begin{array}{l}\text { Flexibility, } \\
\text { strengthening, } \\
\text { cardiovascular } \\
\text { training. }\end{array}$ & $\begin{array}{l}\text { Self-selected gait } \\
\text { speed and strength. }\end{array}$ & Haemodialysis \\
\hline $\begin{array}{l}\text { Chang } \\
2017 \text { [47] }\end{array}$ & Tai Chi. & $\begin{array}{l}\text { Self-selected gait } \\
\text { speed, physical } \\
\text { functioning, } \\
\text { quality of life. }\end{array}$ & Haemodialysis \\
\hline $\begin{array}{l}\text { Headley } \\
2002[53]\end{array}$ & Strengthening. & $\begin{array}{l}\text { Strength and } \\
\text { normal gait speed. }\end{array}$ & Haemodialysis \\
\hline $\begin{array}{l}\text { Painter } \\
2000[57]\end{array}$ & $\begin{array}{l}\text { Flexibility, } \\
\text { strengthening, } \\
\text { cardiovascular } \\
\text { training. }\end{array}$ & $\begin{array}{l}\text { Self-selected and } \\
\text { maximal gait speed. }\end{array}$ & Haemodialysis \\
\hline $\begin{array}{l}\text { Rossi } \\
2014 \text { [45] }\end{array}$ & $\begin{array}{l}\text { Physical therapy } \\
\text { or cardiovascular } \\
\text { rehabilitation. }\end{array}$ & $\begin{array}{l}\text { Self-selected gait } \\
\text { speed, physical } \\
\text { capacity, quality } \\
\text { of life. }\end{array}$ & CKD 3-4 \\
\hline $\begin{array}{l}\text { Storer } \\
2005[49]\end{array}$ & $\begin{array}{l}\text { Cardiovascular } \\
\text { training. }\end{array}$ & $\begin{array}{l}\text { Cardiopulmonary } \\
\text { fitness, strength, } \\
\text { physical function, } \\
\text { gait speed. }\end{array}$ & Haemodialysis \\
\hline $\begin{array}{l}\text { Tao } \\
2015[50]\end{array}$ & $\begin{array}{l}\text { Flexibility, } \\
\text { strengthening, } \\
\text { cardiovascular } \\
\text { training. }\end{array}$ & $\begin{array}{l}\text { Physical function, } \\
\text { self-perceived health, } \\
\text { self-selected gait speed. }\end{array}$ & Haemodialysis \\
\hline
\end{tabular}

cardiovascular rehabilitation alone demonstrated a positive impact on self-selected gait speed both in HD patients [49] and CKD patients stage 3-4 [45]. The same effect was found in HD patients who underwent physical therapy [45], or performed Tai Chi exercise [47]. One study showed an improved maximal gait speed after kidney transplantation in CKD patients [51].

\section{Discussion}

The quality of the articles investigating gait characteristics in CKD patients, when scored with our adapted checklist [23], was low to moderate. External and internal validity in particular were evaluated with a low score (see Additional file 2).

Regarding gait assessment, our review uncovers a rather narrow view on it in patients with CKD. When we consider consensus guidelines for gait assessment it becomes apparent that a major gap in current clinical and research knowledge lies in the fact that the assessments methodology varies greatly and does not follow standardized procedures [58]. It seems, therefore, justified to question some of the approaches used for gait assessment in people with CKD because many reports fail to specify the assessment protocol in detail, and studies do or do not include acceleration and deceleration phases of walking in their assessment strategies, and only a few research groups investigated spatiotemporal gait characteristics in standardized settings: e.g. under single- and dual-task conditions.

With the exception of two [42, 43], all studies measured gait speed during walking over a defined distance using a stopwatch. This explains the dearth of information about spatiotemporal gait parameters in CKD patients. Most publications measured gait as a marker for physical performance, and not with the aim of determining gait function per se in people with CKD. 
Self-selected walking speed was used in the majority of protocols applied. Three studies measured speed only at maximal walking speed [24, 30, 49]. Only Shin and colleagues were interested in gait quality rather than in performance $[42,43]$. For this purpose they used a pressure sensitive walkway system (Gaitrite) to " ... investigate whether muscle strength is related to gait ... " [43] and to "... examine the DTC ${ }^{1}$ of walking in persons undergoing HD ..." [42]. DTC was not evaluated in CKD patients not on dialysis.

Gait assessment under dual task conditions is of great value in determining the severity of gait disorders $[59,60]$; it helps to differentiate between central nervous system pathologies and peripheral pathologies [61], and has a prognostic value in the risk of fall [62]. Surprisingly, these gait characteristics have not been assessed in people with CKD. It is well known that many gait pathologies may stem from changes in different brain structures [63], and persons with CKD experience large burden of comorbidities, including cerebral small vessel disease [64], that are related to changes in gait [65]. Furthermore, this is a population with identified heightened fall risk. Based on the high prevalence of cerebrovascular disease in people with CKD it seems fair to hypothesize that gait impairments in the CKD population may be mediated by small vessel disease, and possibly explain part of the increased fall rates seen in these patients.

We also noticed that gait assessment applied to CKD patients is mostly limited to the dialytic population, largely neglecting pre-dialytic groups, even if there are suggestions that gait abnormalities already exist in early stages of CKD that lead to heightened risk of falls. In this regard only 9 studies [28-30, 38-41, 45, 66] out of 31 identified publications measured gait in CKD patients in the pre-dialytic stage (CKD 1-4).

All the studies that compared CKD patients with age-matched healthy controls, showed a significantly slower gait speed in the patients, both at self-selected $[25,28,33,34,42,43,46]$ and at maximal walking speed [25, 46]. Gait speed in CKD patients is associated with physical [24, 25, 34, 35], cognitive [38], sensory [18] and metabolic $[29,43]$ capacities. Because all of these factors are influenced by CKD severity, slowing down of walking speed seems a logical consequence as shown in Table 2.

The extensive instrumental gait analysis made on a pressure sensitive walkway confirms the influence of cognitive factors on gait quality in people with CKD $[42,43]$. While spatiotemporal gait parameters like gait speed, cadence and stride length are partially influenced by muscle strength, and can be seen as markers of gait performance, this is not the case for variability, and dual-task cost of gait, which also depends on cognitive factors $[67,68]$ and alteration in brain structures $[63,69]$. It seems from these publications that
HD patients had worse gait variability and dual-task cost of walking compared to control groups. This observation warrants further research into these aspects of gait in people with CKD in various stages of their disease.

Inconsistent results are found in a study where gait speed of controls, CKD stage 5 and HD patients is much higher [28], as compared to values reported in all other studies identified in this review (Additional file 3). Being this the only publication of this review that measured a CKD stage 5 group, Table 2 shows a gait speed in CKD 5 patients similar to the mean of control subjects. The slowest gait speed was measured in a study where HD patients underwent a test battery before and after a dialysis session [44]. Because the studied populations and the used gait test protocol ( $4 \mathrm{~m}$ walk test) of these two publications were not grossly deviant from protocols used in other studies, it is difficult to explain these inconsistencies without further detailed information about the different systems used to assess gait.

Some studies we considered in the review attribute gait changes to diseases accompanying the CKD patients; e.g. diabetes [18, 43], cardiac disease [31] or cognitive impairment [38]; however, the fact that in these same studies the groups free of these comorbidities also showed to have a slower gait speed in comparison to healthy controls, indicates that CKD in itself affects gait, and needs to be considered as an independent risk factor for gait disturbance. The fact that interventional studies described in the review show a positive effect on gait speed of physical therapy training (strengthening, flexibility and cardiovascular training or Tai Chi), or kidney transplantation, hints towards gait changes that are at least partly due to behavioural adaptations, and not solely explainable by CKD.

Gait parameters related to falls are gait speed assessed at self-selected and maximal walking speed [70, 71], stride to stride variability [72-74], and dual-task cost of gait [75-77]. No studies included in this review described a relation between gait performance or gait quality and falls. The higher fall rate of CKD patients on and not on dialysis $[6,7,9,10]$, however, and the deterioration of gait speed performance and quality as described in this review, can be associated with a higher fall risk for CKD patients.

Based on our systematic review, more research is needed for patients with CKD that determines whether and in which stage of the disease changes in spatiotemporal variables become apparent, and whether such change is related to changes in cognition and fall events. We recommend a greater research emphasis on evaluating gait during all CKD stages, and following them using longitudinal study designs.

Spatiotemporal parameters are more sensitive to walking disturbance in clinical populations at higher risk of 
falls [43, 78], giving important information about motor control, planning and movement strategies. The underlying neurobiological mechanisms of gait changes in CKD are inadequately understood and require investigation. A tenable theory is that part of the changes observed in gait may stem from changes in the brain; however, evidence for this relation in people with CKD is currently lacking. In the case of physical health and fall prevention outcomes, there is evidence from the general older population that spatiotemporal variables of gait bear important additional information.

Long-term assessment of gait in longitudinal study settings may get facilitated through the advent of new inertial measurement units based technology [79, 80]. Feasible wearable sensor technologies are available and could be employed in future studies in clinical settings and people with CKD. We recommend development and use of a standardized assessment [81] for the validation of such assessment to facilitate data comparability between research groups and clinical CKD populations.

\section{Future direction for studies investigating gait function in CKD}

This systematic review reveals that the field of research into aspects of locomotor functioning in people with CKD is still in its fledgling state. Gait disorders, fall risk and cognitive decline in haemodialsysis patients have been analyzed in patients during the course of their therapy for at least 2 years. However, little is known about the transitional period these individuals experience from pre-dialysis to dialysis. It is, therefore, unclear whether these patients entered therapy with pre-existing deficiencies or whether these developed due to treatment initiation. Future studies should, therefore, select a sample of pre-dialytic individuals and describe the changes of gait and cognitive functioning from 1 year before to 1 year after HD beginning. Whether there is an association between muscle functioning during locomotion and neural drive should also be assessed. In a first step this could be assessed using a cross-sectional study design where people on haemodialysis are compared with healthy controls. Such a study could for example employ "intramuscular coherence" or "EMG-EMG coherence" analysis, which considers the common synchronized oscillatory drive to a pair of sEMG placed over the same muscle [82], and reflects the neural drive from the motor cortex to the muscles $[82,83]$. Such an approach could substantiate or refute a role of the cortex in locomotor functioning of people with CKD. Whether the type of maintenance haemodialysis plays a role in gait dysfunction is a further intriguing point that may be focussed on. Patients regard the time-consuming nature of haemodialysis as problematic and often fill this time being sedentary and watching tv [84]. It can be hypothesised that this behaviour worsens the physical $[85,86]$ and cognitive [87] functioning of these patients.

\section{Conclusions}

Our review uncovers a rather narrow view on gait assessment in patients with CKD, with the main focus on determining and reporting gait speed, which decreases with increasing CKD severity. Although there is growing evidence from the general older population that spatiotemporal parameters such as gait cycle variability are more sensitive in terms of psychomotor changes, this is currently not considered in CKD. Instrumental gait analysis to measure spatiotemporal parameters in CKD should be further developed and employed in longitudinal study settings, because these parameters are more informative for fall risk assessment. Further studies are required to analyse gait quality in $\mathrm{CKD}$ patients.

\section{Endnotes \\ ${ }^{1}$ Authors' note: Dual-task cost}

\section{Additional files}

Additional file 1: Search strategy (DOCX $13 \mathrm{~kb}$ )

Additional file 2: Results of Downs \& Black checklist for quality assessment (DOCX $18 \mathrm{~kb}$ )

Additional file 3: Summary of gait parameters (DOCX $27 \mathrm{~kb}$ )

\section{Abbreviations}

BMI: Body mass index; CKD: Chronic kidney disease; DTC: Dual-task cost; ESRD: End-stage renal disease; HD: Haemodialysis; LVDD: Left ventricular diastolic disfunction; MCl: Mild cognitive impairment; PRISMA: Preferred reporting items for systematic reviews and meta-analysis

\section{Acknowledgements}

None.

Funding

None.

Availability of data and materials Not applicable.

\section{Authors' contributions}

DZ designed the search strategy, extracted data, analysed data and drafted the first version of the manuscript. EdB designed the search strategy, analysed data, supported drafting of the manuscript and critically revised the first version of the manuscript for important intellectual content. OG and PQ contributed to analysis and interpretation of data, and critically revised the manuscript. All authors read and gave final approval of the version to be published, and agree to be accountable for all aspects of the work.

Ethics approval and consent to participate Not applicable.

Consent for publication

Not applicable.

Competing interests

The authors declare that they have no competing interests. 


\section{Publisher's Note}

Springer Nature remains neutral with regard to jurisdictional claims in published maps and institutional affiliations.

\section{Author details \\ ${ }^{1}$ Institute of Human Movement Sciences and Sport, Department of Health Sciences and Technology, ETH Zurich, HCP H 25.1, Leopold-Ruzicka-Weg 4, Zurich, Switzerland. ${ }^{2}$ Geriatric Service, Ente Ospedaliero Cantonale (EOC), Regional Hospital of Mendrisio, Via Turconi 23, 6850 Mendrisio, Switzerland. ${ }^{3}$ Department of Internal Medicine, Ente Ospedaliero Cantonale (EOC), Regional Hospital of Mendrisio, Mendrisio, Switzerland. ${ }^{4}$ Nephrology Service, Switzerland. ${ }^{5}$ Division of Physiotherapy, Department of Neurobiology, Care Sciences and Society, Karolinska Institute, Stockholm, Sweden. ${ }^{6}$ Department of Internal Medicine and Nephrology Service, Ente Ospedaliero Cantonale (EOC), Regional Hospital of Mendrisio, Via Turconi 23, 6850 Mendrisio, Switzerland. \\ Received: 7 December 2018 Accepted: 26 February 2019 \\ Published online: 06 March 2019} Ente Ospedaliero Cantonale (EOC), Regional Hospital of Mendrisio, Mendrisio,

\section{References}

1. Inker LA, Astor BC, Fox CH, Isakova T, Lash JP, Peralta CA, Kurella Tamura M, Feldman HI. KDOQI US commentary on the 2012 KDIGO clinical practice guideline for the evaluation and management of CKD. Am J Kidney Dis. 2014:63(5):713-35.

2. National Kidney F. K/DOQI clinical practice guidelines for chronic kidney disease: evaluation, classification, and stratification. Am J Kidney Dis. 2002; 39(2 Suppl 1):S1-266.

3. G. B. D. CoDC. Global, regional, and national age-sex specific mortality for 264 causes of death, 1980-2016: a systematic analysis for the global burden of disease study 2016. Lancet. 2017;390(10100):1151-210.

4. Jager KJ, Fraser SDS. The ascending rank of chronic kidney disease in the global burden of disease study. Nephrol Dial Transplant. 2017; 32(suppl_2):ii121-8.

5. Jha V, Garcia-Garcia G, Iseki K, Li Z, Naicker S, Plattner B, Saran R, Wang AY, Yang CW. Chronic kidney disease: global dimension and perspectives. Lancet. 2013;382(9888):260-72.

6. Abdel-Rahman EM, Turgut F, Turkmen K, Balogun RA. Falls in elderly hemodialysis patients. Qjm. 2011;104(10):829-38.

7. Cook WL, Tomlinson G, Donaldson M, Markowitz SN, Naglie G, Sobolev B, Jassal SV. Falls and fall-related injuries in older dialysis patients. Clin J Am Soc Nephrol. 2006;1(6):1197-204.

8. Kannus $\mathrm{P}$, Sievanen $\mathrm{H}$, Palvanen $\mathrm{M}$, Jarvinen $\mathrm{T}$, Parkkari J. Prevention of falls and consequent injuries in elderly people. Lancet. 2005;366(9500):1885-93.

9. Kistler BM, Khubchandani J, Jakubowicz G, Wilund K, Sosnoff J. Falls and fallrelated injuries among US adults aged 65 or older with chronic Kidney disease. Prev Chronic Dis. 2018:15:E82.

10. Robertson L, Black C, Fluck N, Gordon S, Hollick R, Nguyen H, Prescott G, Marks A. Hip fracture incidence and mortality in chronic kidney disease: the GLOMMS-II record linkage cohort study. BMJ Open. 2018;8(4):e020312.

11. Plantinga LC, Patzer RE, Franch HA, Bowling CB. Serious fall injuries before and after initiation of hemodialysis among older ESRD patients in the United States: a retrospective cohort study. Am J Kidney Dis. 2017;70(1):76-83.

12. Bridenbaugh SA, Kressig RW. Laboratory review: the role of gait analysis in seniors' mobility and fall prevention. Gerontology. 2011;57(3):256-64.

13. Quadri P, Tettamanti M, Bernasconi S, Trento F, Loew F. Lower limb function as predictor of falls and loss of mobility with social repercussions one year after discharge among elderly inpatients. Aging Clin Exp Res. 2005;17(2):82-9.

14. Merlo A, Zemp D, Zanda E, Rocchi S, Meroni F, Tettamanti M, Recchia A, Lucca U, Quadri P. Postural stability and history of falls in cognitively able older adults: the Canton Ticino study. Gait Posture. 2012;36(4):662-6.

15. Woollacott M, Shumway-Cook A. Attention and the control of posture and gait: a review of an emerging area of research. Gait Posture. 2002;16(1):1-14.

16. Yogev-Seligmann G, Rotem-Galili Y, Mirelman A, Dickstein R, Giladi N, Hausdorff JM. How does explicit prioritization alter walking during dual-task performance? Effects of age and sex on gait speed and variability. Phys Ther. 2010;90(2):177-86.
17. Painter $P$, Marcus R. Physical function and gait speed in patients with chronic kidney disease. Nephrol Nurs J. 2013:40(6):529-38 quiz 539.

18. Jin SH, Park YS, Park YH, Chang HJ, Kim SR. Comparison of gait speed and peripheral nerve function between chronic Kidney disease patients with and without diabetes. Ann Rehabil Med. 2017;41(1):72-9.

19. American Geriatrics Society, British Geriatrics Society, American Academy of Orthopaedic Surgeons Panel on Falls Prevention. Guideline for the prevention of falls in older persons. American Geriatrics Society, British Geriatrics Society, and American Academy of Orthopaedic Surgeons Panel on Falls Prevention. J Am Geriatr Soc. 2001;49(5):664-72.

20. Deandrea S, Lucenteforte E, Bravi F, Foschi R, La Vecchia C, Negri E. Risk factors for falls in community-dwelling older people: a systematic review and meta-analysis. Epidemiology. 2010;21(5):658-68.

21. Liberati A, Altman DG, Tetzlaff J, Mulrow C, Gotzsche PC, loannidis JP, Clarke M, Devereaux PJ, Kleijnen J, Moher D. The PRISMA statement for reporting systematic reviews and meta-analyses of studies that evaluate health care interventions: explanation and elaboration. PLoS Med. 2009;6(7):e1000100.

22. Moher D, Liberati A, Tetzlaff J, Altman DG, Group P. Preferred reporting items for systematic reviews and meta-analyses: the PRISMA statement. PLoS Med. 2009;6(7):e1000097.

23. Downs $\mathrm{SH}$, Black N. The feasibility of creating a checklist for the assessment of the methodological quality both of randomised and non-randomised studies of health care interventions. J Epidemiol Community Health. 1998; 52(6):377-84.

24. Abe $Y$, Matsunaga A, Matsuzawa R, Kutsuna T, Yamamoto S, Yoneki K, Harada M, Ishikawa R, Watanabe T, Yoshida A. Determinants of slow walking speed in ambulatory patients undergoing maintenance hemodialysis. PLoS One. 2016;11(3):e0151037.

25. Bohannon RW, Hull D, Palmeri D. Muscle strength impairments and gait performance deficits in kidney transplantation candidates. Am J Kidney Dis. 1994;24(3):480-5.

26. Bohannon RW, Smith J, Hull D, Palmeri D, Barnhard R. Deficits in lower extremity muscle and gait performance among renal transplant candidates. Arch Phys Med Rehabil. 1995;76(6):547-51.

27. Broers NJH, Martens RJH, Cornelis T, Diederen NMP, Wabel P, van der Sande FM, Leunissen KML, Kooman JP. Body composition in dialysis patients: a functional assessment of bioimpedance using different prediction models. J Ren Nutr. 2015:25(2):121-8.

28. Broers NJH, Martens RJH, Ornelis T, Van Der Sande FM, Diederen NMP, Hermans MMH, Wirtz JJJM, Stifft F, Konings CJAM, Dejagere T, et al. Physical activity in end-stage renal disease patients: the effects of starting Dialysis in the first 6 months after the transition period. Nephron. 2017;137(1):47-56.

29. Gordon PL, Doyle JW, Johansen KL. Association of 1,25-dihydroxyvitamin D levels with physical performance and thigh muscle cross-sectional area in chronic kidney disease stage 3 and 4. J Ren Nutr. 2012;22(4):423-33.

30. Hiraki K, Yasuda T, Hotta C, Izawa KP, Morio Y, Watanabe S, Sakurada T, Shibagaki Y, Kimura K. Decreased physical function in pre-dialysis patients with chronic kidney disease. Clin Exp Nephrol. 2013;17(2):225-31.

31. Jeong JH, Wu P-T, Kistler BM, Fitschen PJ, Biruete AG, Phillips SA, Ali MM, Fernhall B, Wilund KR. The presence and impact of diastolic dysfunction on physical function and body composition in hemodialysis patients. J Nephrol. 2015;28(6):739-47

32. Johansen KL, Chertow GM, da Silva M, Carey S, Painter P. Determinants of physical performance in ambulatory patients on hemodialysis. Kidney Int. 2001;60(4):1586-91.

33. Johansen KL, Painter P, Kent-Braun JA, Ng AV, Carey S, Da Silva M, Chertow GM. Validation of questionnaires to estimate physical activity and functioning in end-stage renal disease. Kidney Int. 2001;59(3):1121-7.

34. Johansen KL, Shubert T, Doyle J, Soher B, Sakkas GK, Kent-Braun JA. Muscle atrophy in patients receiving hemodialysis: effects on muscle strength, muscle quality, and physical function. Kidney Int. 2003;63(1):291-7.

35. Kittiskulnam P, Carrero JJ, Chertow GM, Kaysen GA, Delgado C, Johansen KL. Sarcopenia among patients receiving hemodialysis: weighing the evidence. J Cachexia Sarcopenia Muscle. 2017:8(1):57-68.

36. Kittiskulnam P, Chertow GM, Carrero JJ, Delgado C, Kaysen GA, Johansen KL. Sarcopenia and its individual criteria are associated, in part, with mortality among patients on hemodialysis. Kidney Int. 2017;92(1):238-47.

37. Kutsuna T, Matsunaga A, Matsumoto T, Ishii A, Yamamoto K, Hotta K, Aiba N, Takagi Y, Yoshida A, Takahira N, et al. Physical activity is necessary to prevent deterioration of the walking ability of patients undergoing maintenance hemodialysis. Ther Apher Dial. 2010;14(2):193-200. 
38. Otobe Y, Hiraki K, Hotta C, Nishizawa H, Izawa KP, Taki Y, Imai N, Sakurada T, Shibagaki Y. Mild cognitive impairment in older adults with pre-dialysis patients with chronic kidney disease: Prevalence and association with physical function. Nephrology (Carlton, Vic). 2017.

39. Padilla J, Krasnoff J, Da Silva M, Hsu CY, Frassetto L, Johansen KL, Painter P. Physical functioning in patients with chronic kidney disease. J Nephrol. 2008;21(4):550-9.

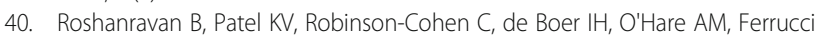
L, Himmelfarb J, Kestenbaum B. Creatinine clearance, walking speed, and muscle atrophy: a cohort study. Am J Kidney Dis. 2015;65(5):737-47.

41. Roshanravan B, Robinson-Cohen C, Patel KV, Ayers E, Littman AJ, de Boer $\mathbb{H}$, Ikizler TA, Himmelfarb J, Katzel LI, Kestenbaum B, et al. Association between physical performance and all-cause mortality in CKD. J Am Soc Nephrol. 2013;24(5):822-30.

42. Shin S, Chung HR, Kistler BM, Fitschen PJ, Wilund KR, Sosnoff JJ. Walking and talking in maintenance hemodialysis patients. Arch Phys Med Rehabil. 2013;94(1):127-31.

43. Shin S, Chung HR, Kistler BM, Fitschen PJ, Wilund KR, Sosnoff JJ. Effect of muscle strength on gait in hemodialysis patients with and without diabetes. Int J Rehabil Res. 2014;37(1):29-33.

44. Wolfgram DF, Lathara Z, Szabo A, Whittle J. Dialytic hemodynamics are associated with changes in gait speed. Hemodial Int. 2016.

45. Rossi AP, Burris DD, Lucas FL, Crocker GA, Wasserman JC. Effects of a renal rehabilitation exercise program in patients with CKD: a randomized, controlled trial. Clin J Am Soc Nephrol. 2014;9(12):2052-8.

46. Blake C, O'Meara YM. Subjective and objective physical limitations in highfunctioning renal dialysis patients. Nephrol Dial Transplant. 2004;19(12):3124-9.

47. Chang JH, Koo M, Wu SW, Chen CY. Effects of a 12-week program of tai chi exercise on the kidney disease quality of life and physical functioning of patients with end-stage renal disease on hemodialysis. Complement Ther Med. 2017;30:79-83.

48. Painter P, Carlson L, Carey S, Paul SM, Myll J. Physical functioning and health-related quality-of-life changes with exercise training in hemodialysis patients. Am J Kidney Dis. 2000;35(3):482-92.

49. Storer TW, Casaburi R, Sawelson S, Kopple JD. Endurance exercise training during haemodialysis improves strength, power, fatigability and physical performance in maintenance haemodialysis patients. Nephrol Dial Transplant. 2005;20(7):1429-37.

50. Tao X, Chow SKY, Wong FKY. A nurse-led case management program on home exercise training for hemodialysis patients: a randomized controlled trial. Int J Nurs Stud. 2015;52(6):1029-41.

51. Bohannon RW, Smith J, Hull D, Palmeri D, Barnhard R. Strength, balance and gait before and after kidney transplantation. Int J Rehabil Res. 1997;20(2) 199-203.

52. Cappy CS, Jablonka J, Schroeder ET. The effects of exercise during hemodialysis on physical performance and nutrition assessment. J Ren Nutr. 1999;9(2):63-70.

53. Headley S, Germain M, Mailloux P, Mulhern J, Ashworth B, Burris J, Brewer B, Nindl BC, Coughlin M, Welles R, et al. Resistance training improves strength and functional measures in patients with end-stage renal disease. Am J Kidney Dis. 2002;40(2):355-64.

54. Trac MH, McArthur E, Jandoc R, Dixon SN, Nash DM, Hackam DG, Garg AX. Macrolide antibiotics and the risk of ventricular arrhythmia in older adults. CMAJ. 2016;188(7):E120-9.

55. Landis JR, Koch GG. The measurement of observer agreement for categorical data. Biometrics. 1977;33(1):159-74.

56. Hausdorff JM, Schweiger A, Herman T, Yogev-Seligmann G, Giladi N. Dualtask decrements in gait: contributing factors among healthy older adults. J Gerontol A Biol Sci Med Sci. 2008;63(12):1335-43.

57. Painter P, Carlson L, Carey S, Paul SM, Myll J. Low-functioning hemodialysis patients improve with exercise training. Am J Kidney Dis. 2000;36(3):600-8.

58. Beauchet $\mathrm{O}$, Allali G, Sekhon H, Verghese J, Guilain S, Steinmetz JP, Kressig RW, Barden JM, Szturm T, Launay CP, et al. Guidelines for assessment of gait and reference values for spatiotemporal gait parameters in older adults: the Biomathics and Canadian gait consortiums initiative. Front Hum Neurosci. 2017;11:353

59. Ellmers TJ, Cocks AJ, Doumas M, Williams AM, Young WR. Gazing into thin air: the dual-task costs of movement planning and execution during adaptive gait. PLoS One. 2016;11(11):e0166063.

60. Montero-Odasso M, Verghese J, Beauchet O, Hausdorff JM. Gait and cognition: a complementary approach to understanding brain function and the risk of falling. J Am Geriatr Soc. 2012;60(11):2127-36.
61. Auvinet B, Touzard C, Montestruc F, Delafond A, Goeb V. Gait disorders in the elderly and dual task gait analysis: a new approach for identifying motor phenotypes. J Neuroeng Rehabil. 2017;14(1):7.

62. Muir-Hunter SW, Wittwer JE. Dual-task testing to predict falls in communitydwelling older adults: a systematic review. Physiotherapy. 2016;102(1):29-40.

63. Tian Q, Chastan N, Bair WN, Resnick SM, Ferrucci L, Studenski SA. The brain map of gait variability in aging, cognitive impairment and dementia-A systematic review. Neurosci Biobehav Rev. 2017;74(Pt A):149-62.

64. Weiner DE, Seliger SL. Cognitive and physical function in chronic kidney disease. Curr Opin Nephrol Hypertens. 2014;23(3):291-7.

65. van der Holst HM, Tuladhar AM, Zerbi V, van Uden IWM, de Laat KF, van Leijsen EMC, Ghafoorian M, Platel B, Bergkamp MI, van Norden AGW, et al. White matter changes and gait decline in cerebral small vessel disease. Neuroimage Clin. 2018;17:731-8.

66. Bohannon RW. Comfortable and maximum walking speed of adults aged 20 79 years: reference values and determinants. Age Ageing. 1997;26(1):15-9.

67. Beauchet O, Annweiler C, Montero-Odasso M, Fantino B, Herrmann FR, Allali G. Gait control: a specific subdomain of executive function? J Neuroeng Rehabil. 2012;9:12

68. Coppin AK, Shumway-Cook A, Saczynski JS, Patel KV, Ble A, Ferrucci L, Guralnik JM. Association of executive function and performance of dual-task physical tests among older adults: analyses from the InChianti study. Age Ageing. 2006;35(6):619-24.

69. Rosso AL, Olson Hunt MJ, Yang M, Brach JS, Harris TB, Newman AB, Satterfield S, Studenski SA, Yaffe K, Aizenstein HJ, et al. Higher step length variability indicates lower gray matter integrity of selected regions in older adults. Gait Posture. 2014;40(1):225-30.

70. Middleton A, Fulk GD, Herter TM, Beets MW, Donley J, Fritz SL. Self-selected and maximal walking speeds provide greater insight into fall status than walking speed reserve among community-dwelling older adults. Am J Phys Med Rehabil. 2016;95(7):475-82.

71. Quach L, Galica AM, Jones RN, Procter-Gray E, Manor B, Hannan MT, Lipsitz $L A$. The nonlinear relationship between gait speed and falls: the maintenance of balance, independent living, intellect, and zest in the elderly of Boston study. J Am Geriatr Soc. 2011;59(6):1069-73.

72. Brach JS, Berlin JE, VanSwearingen JM, Newman AB, Studenski SA. Too much or too little step width variability is associated with a fall history in older persons who walk at or near normal gait speed. J Neuroeng Rehabil. 2005;2:21

73. Callisaya ML, Blizzard L, Schmidt MD, Martin KL, McGinley JL, Sanders LM, Srikanth VK. Gait, gait variability and the risk of multiple incident falls in older people: a population-based study. Age Ageing. 2011;40(4):481-7.

74. Hausdorff JM, Rios DA, Edelberg HK. Gait variability and fall risk in community-living older adults: a 1-year prospective study. Arch Phys Med Rehabil. 2001;82(8):1050-6.

75. Al-Yahya E, Dawes H, Smith L, Dennis A, Howells K, Cockburn J. Cognitive motor interference while walking: a systematic review and meta-analysis. Neurosci Biobehav Rev. 2011;35(3):715-28.

76. Lundin-Olsson L, Nyberg L, Gustafson Y. "Stops walking when talking" as a predictor of falls in elderly people. Lancet. 1997;349(9052):617.

77. Mirelman A, Herman T, Brozgol M, Dorfman M, Sprecher E, Schweiger A, Giladi N, Hausdorff JM. Executive function and falls in older adults: new findings from a five-year prospective study link fall risk to cognition. PLoS One. 2012;7(6):e40297.

78. Lockhart TE, Barth AT, Zhang X, Songra R, Abdel-Rahman E, Lach J. Portable, non-invasive fall risk assessment in end stage renal disease patients on hemodialysis. ACM Trans Comput Hum Interact. 2010:84-93.

79. Allet $L$, Knols RH, Shirato K, de Bruin ED. Wearable systems for monitoring mobility-related activities in chronic disease: a systematic review. Sensors (Basel). 2010;10(10):9026-52.

80. de Bruin ED, Hartmann A, Uebelhart D, Murer K, Zijlstra W. Wearable systems for monitoring mobility-related activities in older people: a systematic review. Clin Rehabil. 2008;22(10-11):878-95.

81. Jamour M, Becker C, Bachmann S, de Bruin ED, Gruneberg C, Heckmann J, Marburger C, Nicolai SE, Schwenk M, Lindemann U. Recommendation of an assessment protocol to describe geriatric inpatient rehabilitation of lower limb mobility based on ICF: an interdisciplinary consensus process. Z Gerontol Geriatr. 2011:44(6):429-36.

82. Hansen NL, Hansen S, Christensen LO, Petersen N, Nielsen JB. Synchronization of lower limb motor unit activity during walking in human subjects. J Neurophysiol. 2001;86(3):1266-76. 
83. Gennaro F, de Bruin ED. Assessing brain-muscle connectivity in human locomotion through Mobile brain/body imaging: opportunities, pitfalls, and future directions. Front Public Health. 2018;6:39.

84. Borzou SR, Anosheh M, Mohammadi E, Kazemnejad A. Patients' perception of comfort facilitators during hemodialysis procedure: a qualitative study. Iran Red Crescent Med J. 2014;16(7):e19055.

85. Anderton N, Giri A, Wei G, Marcus RL, Chen X, Bjordahl T, Habib A, Herrera J, Beddhu S. Sedentary behavior in individuals with diabetic chronic Kidney disease and maintenance hemodialysis. J Ren Nutr. 2015;25(4):364-70.

86. DiPietro L, Jin Y, Talegawkar S, Matthews CE. The joint associations of sedentary time and physical activity with mobility disability in older people: the NIH-AARP diet and health study. J Gerontol A Biol Sci Med Sci. 2018; 73(4):532-8.

87. Polinder-Bos HA, Garcia DV, Kuipers J, Elting JWJ, Aries MJH, Krijnen WP, Groen H, Willemsen ATM, van Laar PJ, Strijkert F, et al. Hemodialysis induces an acute decline in cerebral blood flow in elderly patients. J Am Soc Nephrol. 2018;29(4):1317-25.

Ready to submit your research? Choose BMC and benefit from:

- fast, convenient online submission

- thorough peer review by experienced researchers in your field

- rapid publication on acceptance

- support for research data, including large and complex data types

- gold Open Access which fosters wider collaboration and increased citations

- maximum visibility for your research: over $100 \mathrm{M}$ website views per year

At $\mathrm{BMC}$, research is always in progress.

Learn more biomedcentral.com/submissions 